\title{
Thermal Radiation of Hydromagnetic Stagnation Gravity-Driven Flow through a Porous Confined Cylinder with Non-Uniform Heat Source
}

\author{
O. K. Onanuga ${ }^{1}$, M. A. C. Chendo ${ }^{2}$, N. E. Erusiafe ${ }^{2}$ \\ ${ }^{1}$ Department of Physics, Lagos State Polytechnic, Ikorodu, Lagos State, Nigeria \\ ${ }^{2}$ Department of Physics, University of Lagos, Akoka, Lagos State, Nigeria \\ Email: *onanuga.olutayo@gmail.com
}

How to cite this paper: Onanuga, O.K., Chendo, M.A.C. and Erusiafe, N.E. (2018) Thermal Radiation of Hydromagnetic Stagnation Gravity-Driven Flow through a Porous Confined Cylinder with Non-Uniform Heat Source. Open Journal of Fluid Dynamics, 8, 361-377.

https://doi.org/10.4236/ojfd.2018.84023

Received: August 7, 2018

Accepted: November 5, 2018

Published: November 8, 2018

Copyright $\odot 2018$ by authors and Scientific Research Publishing Inc. This work is licensed under the Creative Commons Attribution International License (CC BY 4.0).

http://creativecommons.org/licenses/by/4.0/

\begin{abstract}
The focus of the study is to examine thermal radiation and viscous dissipative heat transfers of magnetohydrodynamics (MHD) stagnation point flow past a permeable confined stretching cylinder with non-uniform heat source or sink. The formulated equation governing the flow is non-dimensional. The dimensionless momentum and energy equation are solved using shooting technique coupled with fourth-order Runge-kutta integrated scheme which satisfied smoothness conditions at the edge of the boundary layer. The result for the velocity and temperature distributions are presented graphically and discussed to portray the effects of some important embodiment parameters on the flow. The Nusselt number and skin friction were obtained and compared with the previous scholars' results in others to validate the present research work.
\end{abstract}

\section{Keywords}

Dissipation, Stagnation Flow, Magnetohydrodynamic Flow, Thermal Radiation, Heat Source/Sink

\section{Introduction}

The science dealing with the motion of electrically conducting fluids through magnetic fields is known as magnetohydrodynamics. Fluid mechanics and magnetic theory are harmonized as magnetohydrodynamics (MHD), the field which was initiated in [1]. Magnetohydrodynamics is the multi-disciplinary finding of fluid flow of an electrically conducting fluid through magnetic field which can 
subjectively divide into plasma physics. The feedback of MHD stagnation flow of heat transfer in a cylinder through a boundary layer near a permeable plate in the presence of non-uniform heat source is important from the technical point of view. The MHD has received a considerable amount of special treatment due to its instantaneous applications in several devices such as accelerators, MHD power generators, MHD pumps and others [2]. Recently, the desires of contemporary technology have inspired the attention of many scientists in the studies of fluid flow that involve the interaction of many phenomena. The study includes stagnation point flow via a porous surface which plays an imperative role in so many technical problems such as the production of friendly environment stand-alone power generator from the principle of Tesla bladeless turbine and boundary layer condition. However, an analysis for heat transfer in a hydromagnetic flow via a stretching surface [3] with, the investigation on heat transfer for viscous dissipation effects on the external magnetic field was done in the works of (Abel et al.) [4]. The MHD flow heat transfer of fluid-metal in circular pipes for non-heated cases and externally heated was considered [5]. The study of a transient convection flow over a permeable stretching plate and heat transfer with thermal radiation was considered by (Mukhopadhyay et al.). More so, the influences of variable viscosity on thermal diffusivity boundary layer flow over a permeable broadening surface presented in [6].

\section{Previous Work on Magnetohydrodynamic and Related Issues}

The study of chemical reaction and viscous dissipation for free convective hydromagnetic flow past an inclined porous stretching surface was reported in the works of (Alam, M.S) [7]. In the study, the influence of Eckert number Ec was reported to have significant effects on the heat and momentum distribution. The analysis [8] of thermal radiation effects on motioning semi-infinite vertical cylinder in an MHD flow with heat transfer was reported. The reaction of a non-uniform heat source on liquid heat transfer past a porous stretching sheet was verified in [9] and [10]. Several scientists have carried out related studies for power-law non-Newtonian fluids Chen [11] [12] [13]. Many studies were carried out on cylindrical geometry [14] [15] [16]. The influence of magnetic field and injection/suction of heat transfer on a laminar fluid flow through a stretching cylinder in the absence of transverse curvature is presented in (Ishak, A et al.) [17]. The transient flow of heat and species transfer past a decrease cylinder was examined in the works of (Wan Zaimi et al.) [18]. Heat transfer of MHD stagnation point flow [19] over a porous plate was presented. Meanwhile, the dissipation and radiation effect on thermal conductivity and variable viscosity hydromagnetic heat transfer near a stagnation point flow reported in (Salawu, S.O et al.) [20]. The computational results of the study were obtained using Runge-Kutta method. In the study, it was reported that the magnetic field parameter decreases the flow while thermal Grashof parameter raises the flow velocity. The trans- 
verse curvature parameter can be defined as the ratio of the boundary layer thickness to the cylinder radius. At lower transverse curvature parameters, the model equations maybe expressed in a two-dimensional form. For a slim cylinder, nevertheless, the problem develops to axisymmetric; when the outer surface transverse curvature parameter occurs in the formulated model. A similarity solution for the model equation of the momentum and heat transfer besides an elongating cylinder [21] suggested it. Later on, [21] reported a computational analysis of the flowing liquid and energy transfer past a parallel cylinder with studying the consequence of the prescribed surface heat flux and transverse curvature parameter. Heat and mass transfer of second-grade fluid in MHD stagnation point flow over an extending cylinder without radiation and heat source/sink was examined [22]. Moreover, in [23] [24] Analyzed heat transfer of MHD stagnation flow past non-isothermal stretching and stationary cylinder. The analysis was done without radiation, viscous dissipation, heat source and thermal buoyancy but [25] reported on the decreasing effect of Reynolds number and magnetic field parameters on the flow velocity profiles in. The effect of the thermal conductivity on the temperature-dependent flow and thermal boundary layer sideways of a broadening cylinder was investigated in (Sharma, et al.) [26]. They examined the magnetic field and external heat source for two diverse thermal boundary situations. Some other scholars carried out studies on mixed convection over a vertical stretching slender cylinder [27] [28]. In the current study, the influences of the magnetic field, thermal buoyancy force, radiation, dissipation and non-uniform heat sink/source with load loss in two dimensional laminar, MHD boundary layer flow of an incompressible viscous electrically conducting fluids past along an axisymmetric, isothermal and moving vertical thick semi-infinite permeable solid cylinder is considered. The mathematical formulation is presented in Section 3 and the fourth order Runge-Kutta integrated scheme along with shooting techniques is employed in space for the computation. In Section 4, the numerical computation with the graphical results is established and quantitatively explained with respect to existing fluid parameters entrenched in the flow.

\section{Mathematical Formulation}

Consider a laminar, two dimensional, hydromagnetic, incompressible viscous fluid flow in an electrically conducting, heated and progressing vertical thin resistant semi-infinite solid cylinder of the radius $R$. The cylindrical coordinates $(z, r)$ are presumed to be the axis of the cylinder which is parallel to the uniform composite free stream flow. In addition, at the center of leading edge of the cylinder is the origin of the coordinate system. A uniform weak magnetic field density $B_{0}$ is applied normal to the flow direction. The cylinder moves linearly with steady velocity about its axis in the same direction as that of the free stream velocity with the temperature $T$ of the free stream fluid. The cylinder surface is maintained at stable temperature $T_{w}$ while $T_{\infty}$ is the ambient temperature (Figure 1). It is assumed that $T_{w}>T_{\infty}$. The physical geometry model, 


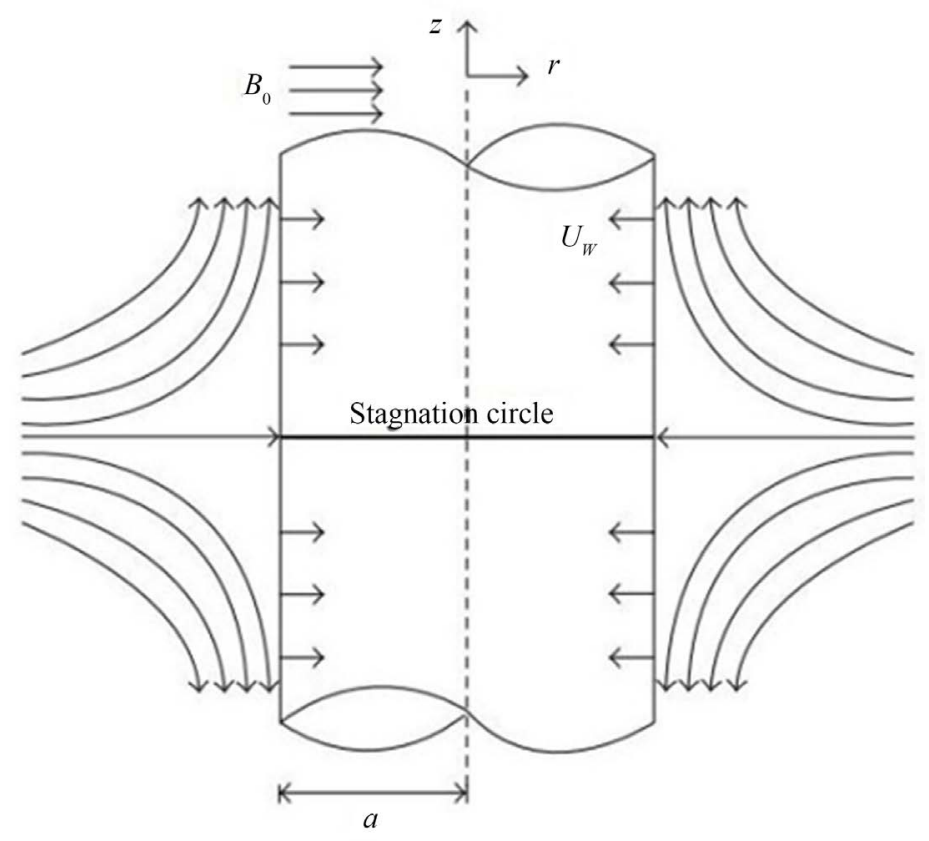

Figure 1. Physical geometry model.

as well as the equations governing the momentum and energy balance, are as follows:

$$
\begin{gathered}
\frac{\partial(r u)}{\partial z}+\frac{\partial(r v)}{\partial r}=0 \\
u \frac{\partial u}{\partial z}+v \frac{\partial u}{\partial r}=-\frac{1}{\rho} \frac{\mathrm{d} P_{e}}{\mathrm{~d} z}+\frac{v}{r} \frac{\partial}{\partial r}\left(r \frac{\partial u}{\partial r}\right)-\frac{\sigma B_{0}^{2} u}{\rho}+g \beta\left(T-T_{\infty}\right)-\frac{m}{r} u^{2} \\
u \frac{\partial T}{\partial z}+v \frac{\partial T}{\partial r}=\frac{k}{\rho c_{p}} \frac{1}{r} \frac{\partial}{\partial r}\left(r \frac{\partial T}{\partial r}\right)-\frac{1}{\rho c_{p}} \frac{1}{r} \frac{\partial}{\partial r}\left(r q_{r}\right)+\frac{v}{c_{p}}\left(\frac{\partial u}{\partial r}\right)^{2}+\frac{q^{\prime \prime \prime}}{\rho c_{p}}
\end{gathered}
$$

Subject to slip boundary conditions

$$
\begin{aligned}
& u(z, r)=U_{w}(z)=\frac{u_{0} z}{a}, v(z, r)=-v_{w}, \\
& T(z, r)=T_{w}(z)=T_{\infty}+\left(\frac{z}{a}\right)^{n} \Delta T \text { at } r=0 \\
& u(z, r)=u_{e}(z)=\frac{u_{\infty} z}{a}, T(z, r)=T_{\infty} \text { at } r \rightarrow \infty
\end{aligned}
$$

From the above, $u$ and $v$ are the velocity elements in the $z$ and $r$ directions respectively also the following parameters $P_{e}, v, \rho, \sigma, B_{0}, g, \beta$ and $m$ represent the elongating pressure gradient, kinematic viscosity, fluid density, electrical conductivity, uniform magnetic field, the acceleration due to gravity, the coefficient of expansivity and fluid mass. $k, c_{p}, q_{r}, u_{0}$ and $u_{\infty}$ stand for the thermal conductivity, specific heat at constant pressure, thermal radiation, stretching velocity and free stream velocity respectively.

The term $\frac{\mathrm{d} P_{e}}{\mathrm{~d} z}$ in Equation (2) can be defined as 


$$
\frac{\mathrm{d} p_{e}}{\mathrm{~d} z}=-\rho u_{e} \frac{\mathrm{d} u_{e}}{\mathrm{~d} z}-\sigma B_{0}^{2} u_{e}
$$

According to Rosseland diffusion approximation for radiation, $q_{r}$ can be defined as (Salawu and Amoo (2016));

$$
q_{r}=-\frac{4 \sigma_{0}}{3 \delta} \frac{\partial T^{4}}{\partial r}
$$

where $\sigma_{0}$ and $\delta$ are the Stefan-Boltzmann and the mean absorption coefficient respectively. Let heat difference in the flow be satisfactory low, $T^{4}$ may be expressed linearly as a function of temperature, applying Taylor series to expand $T^{4}$ is expanded about $T_{\infty}$

$$
T^{4} \cong T_{\infty}^{4}+4 T_{\infty}^{3}\left(T-T_{\infty}\right)+6 T_{\infty}^{2}\left(T-T_{\infty}\right)^{2}+\cdots
$$

Neglecting higher-order terms of $T-T_{\infty}$ in Equation (8) to get

$$
T^{4} \approx 4 T_{\infty}^{3} T-3 T_{\infty}^{4}
$$

Using Equation (8) on Equation (6) to obtain

$$
q_{r}=-\frac{16 T_{\infty}^{3} \sigma_{0}}{3 \delta} \frac{\partial T}{\partial r}
$$

The non-uniform heat source is defined as (Salawu and Fatunmbi (2017))

$$
q^{\prime \prime \prime}=\frac{k u_{w}(z)}{z v}\left[\lambda\left(T-T_{\infty}\right)+\lambda^{*}\left(T_{w}-T_{\infty}\right) \mathrm{e}^{-\eta}\right]
$$

where $\lambda^{*}$ and $\lambda$ denote the heat source and space coefficients temperature dependent respectively while $T_{\infty}$ is the free stream temperature. Here, $\lambda>0$ and $\lambda^{*}>0$ represent heat source but $\lambda<0$ and $\lambda^{*}<0$ depict heat sink.

Using Equation (5), Equation (9) and Equation (10) along with the similarity variables to Equation (11) as defined in Hayat et al. (2017) on Equations (1) to (4) gives;

$$
u=\frac{u_{0} z}{a} f^{\prime}(\eta), v=-\frac{R}{r}\left(\frac{u_{0} v}{a}\right)^{\frac{1}{2}} f(\eta), \theta(\eta)=\frac{T-T_{\infty}}{T_{w}-T_{\infty}}, \psi=\left(\frac{v z^{2} u_{0}}{a}\right)^{\frac{1}{2}} R f(\eta),
$$

Hence, the governing equations and the boundary conditions reduce to the nonlinear coupled ordinary differential equation as follows,

$$
\begin{aligned}
& (1+2 K \eta) f^{\prime \prime \prime}(\eta)+2 K f^{\prime \prime}(\eta)+f(\eta) f^{\prime \prime}(\eta)-(1+Z)\left(f^{\prime}(\eta)\right)^{2} \\
& -H^{2}\left(f^{\prime}(\eta)-\gamma\right)+\gamma^{2}+G r \theta(\eta)=0 \\
& \left(1+\frac{4}{3} N\right)\left((1+2 K \eta) \theta^{\prime \prime}(\eta)+2 K \theta^{\prime}(\eta)\right)+\operatorname{Prf}(\eta) \theta^{\prime}(\eta)-n P r f^{\prime}(\eta) \theta(\eta) \\
& +\lambda^{*} \mathrm{e}^{-\eta}+\lambda \theta+E c P r(1+2 K \eta)\left(f^{\prime \prime}(\eta)\right)^{2}=0
\end{aligned}
$$

Subject to the boundary conditions,

$$
\begin{aligned}
& f^{\prime}(\eta)=1, f=f_{w}, \theta=1, \text { at } \eta=0, \\
& f^{\prime}=\gamma, \theta=0, \text { as } \eta \rightarrow \infty
\end{aligned}
$$


where $K=\frac{1}{R} \sqrt{\frac{a v}{u_{0}}}$ is the curvature parameter, $Z=\frac{z m}{r}$ is the load potential difference, $H^{2}=\frac{\sigma B_{0}^{2}}{\rho u_{0}}$ is the Hartman number, $G r=\frac{a^{2} g \beta\left(T_{w}-T_{\infty}\right)}{z u_{0}^{2}}$ is the thermal Grashof number, $\gamma=\frac{u_{e}}{U_{w}}$ is the stagnation rate ratio $\quad N=\frac{4 \sigma_{0} T_{\infty}^{3}}{\delta k}$ is the radiation parameter, $P_{r}=\frac{\rho C_{p}}{k}$ is the Prandtl number, $E c=\frac{u_{0}^{2}}{C_{p}\left(T_{w}-T_{\infty}\right)}$ is the Echert number, $f_{w}=-v_{w} \sqrt{\frac{a}{v u_{0}}}$ is the suction $f_{w}>0$ or injection $f_{w}<0$ parameter.

The engineering interest of the physical quantities are the skin friction and Nusselt number that is $C_{f}$ and $N u$ defined as follow,

$$
C_{f z}=\frac{2 \tau_{w}}{\rho u_{0}^{2}},
$$

where $\tau_{w}$

$$
N_{u z}=\frac{z q_{w}}{k\left(T_{w}-T_{\infty}\right)}
$$

and $q_{w}$ are defined as following

$$
\begin{gathered}
\tau_{w}=\mu\left(\frac{\partial u}{\partial r}\right)_{r=R}=\left(\frac{\partial u}{\partial r}+\left(v \frac{\partial^{2} u}{\partial r^{2}}+u \frac{\partial^{2} u}{\partial z \partial r}\right)\right)_{r=R} \\
q_{w}=-k\left(\frac{\partial T}{\partial r}\right)_{r=R}=-\left(k+\frac{16 \sigma_{0} T_{\infty}^{3}}{3 \delta}\right)\left(\frac{\partial T}{\partial r}\right)_{r=R}
\end{gathered}
$$

using Equation (15) on Equation (17) result to,

$$
R_{e z}^{\frac{1}{2}} C_{f z}=f^{\prime \prime}(0)
$$

Also, using Equation (16) on Equation (18) gives,

$$
R_{e z}^{-\frac{1}{2}} N_{u z}=-\left(1+\frac{4}{3} N\right) \theta^{\prime}(0)
$$

where $R_{e z}=\frac{z u_{0}}{v}$ shows the local Reynolds number (Table 1 and Table 2).

\section{Results and Discussion}

The set of non-linear differential equations alongside the boundary conditions are unraveled numerically by applying fourth-order Runge-Kutta integration technique couple with shooting method. The smoothness conditions are confirmed through checking at the edge of the boundary layer whether they are satisfied. Calculations are conducted for different values of the following default parameters based on existing research works, the following diverse computations values as set as the default values for the embedded parameters; $f^{\prime \prime}(0)$ for $K=0, H=0$, 
Table 1. Comparison of $f^{\prime \prime}(0)$ for $K=0, H=0, G r=0, Z=0, \lambda=0, n=0$, $\lambda^{*}=0, \quad N=0, f_{w}=0, \quad \operatorname{Pr}=1, E c=0.2, \quad \eta \rightarrow \infty$.

\begin{tabular}{ccccc}
\hline$\gamma$ & Pop et al. (2004) [29] & Sharma (2009) [26] & Hayat et al. (2014) [30] & Present results \\
\hline 0.1 & -0.9694 & -0.969386 & -0.9679 & -0.9694 \\
0.2 & -0.9181 & -0.9181069 & -0.9172 & -0.9181 \\
0.5 & -0.6673 & -0.667263 & -0.6670 & -0.6673 \\
2.0 & 2.0174 & 2.01749079 & 2.0174 & 2.0175 \\
3.0 & 4.7293 & 4.72922695 & 4.7294 & 4.7293 \\
\hline
\end{tabular}

Table 2. Comparison of $\theta^{\prime}(0)$ for $K=0, H=0, G r=0, Z=0, \gamma=0, \lambda=0$, $\lambda^{*}=0, \quad N=0, f_{w}=0, \quad \operatorname{Pr}=1, E c=0.2, \quad \eta \rightarrow \infty$.

\begin{tabular}{cccccc}
\hline$P r$ & $n$ & Mukhopadhyay (2012) [27] & Pal (2012) [28] Hayat et al. (2014) [30] & Present results \\
\hline 1 & -2 & - & - & -1.0000 & -0.9889 \\
& -1 & - & - & -0.0 & -0.0025 \\
& 0 & 0.5821 & - & -0.5832 & -0.5826 \\
& 1 & 1.0000 & - & 1.0000 & 1.0002 \\
& 2 & 1.3332 & 1.333333 & 1.3332 & 1.3333 \\
10 & -2 & - & - & 10.0000 & 9.9964 \\
& -1 & - & - & 0.0 & 0.0 \\
& 0 & - & - & 2.3080 & 2.3080 \\
& 1 & - & - & 3.7207 & 3.7206 \\
& 2 & - & -1.796871 & 4.7969 & 4.7968 \\
0.7 & 2 & - & - & 1.0791 & 1.0702 \\
0.6 & 2 & - & - & - & 0.9708 \\
0.5 & 2 & - & - & - & 0.8637 \\
\hline
\end{tabular}

$G r=0, Z=0, \lambda=0, n=0, \lambda^{*}=0, \quad N=0, f_{w}=0, \quad P r=1, E c=0.2$, $\eta \rightarrow \infty$. and $\theta^{\prime}(0)$ for $K=0, H=0, G r=0, Z=0, \gamma=0, \lambda=0$, $\lambda^{*}=0, N=0, f_{w}=0, \operatorname{Pr}=1, E c=0.2, \eta \rightarrow \infty$.

All graphs satisfy the values except otherwise stated in the respective graph.

Figure 2 depicts the effect of various values of magnetic field parameter $H$ on fluid flow velocity. It is observed that the rate of fluid motion is considerably reduced with an increase in the $\mathrm{H}$ values. The damping magnetic properties rise due to the presence of the Lorentz force that results in increased resistance to flow and thereby reduced the convective fluid flow motion. Also, the electrically conducting liquid takes a thrust from the magnetic force by inducing an electrically conducting fluid in the microscale system making it warmer as it progresses along the plate by leading to a reduction in the velocity profiles.

The impact of diverse values of the thermal Grashof number $G r$ on the momentum distributions is shown in Figure 3. It was observed that changes in 


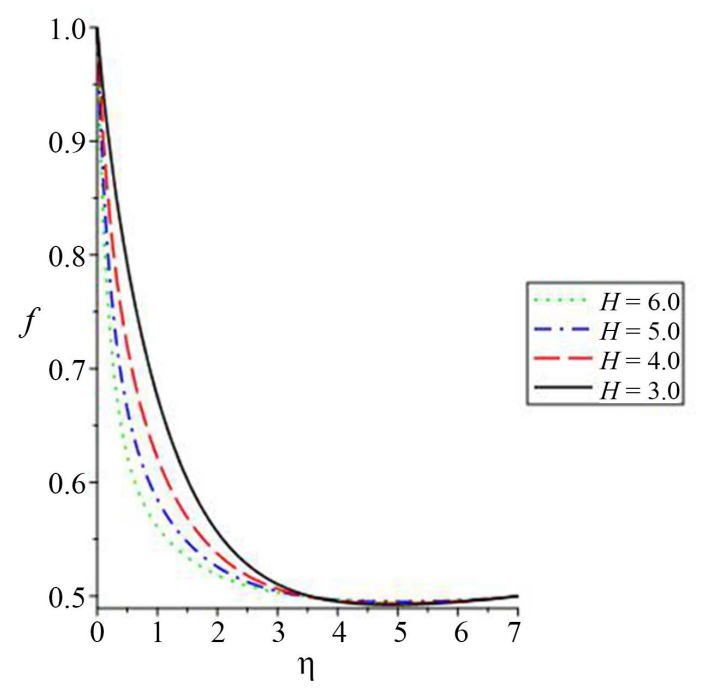

Figure 2.Velocity Profiles for values of $H$.

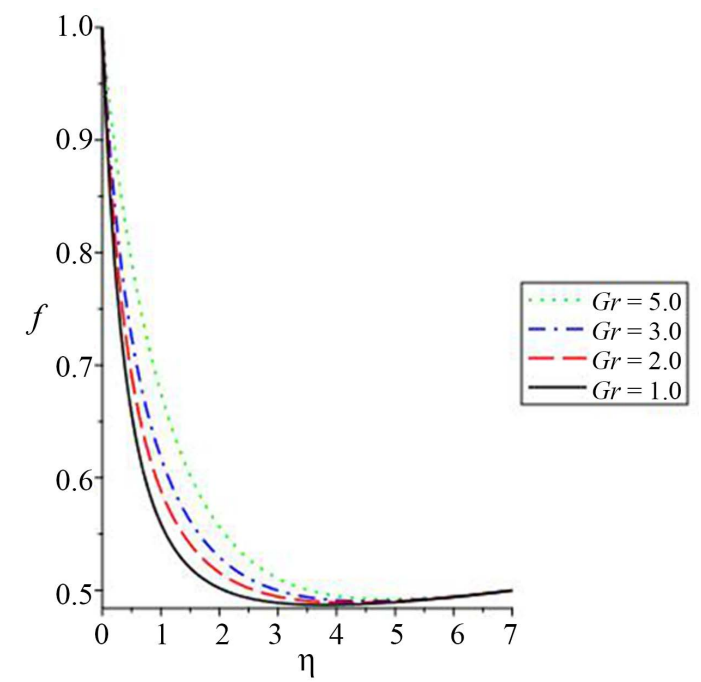

Figure 3. Velocity Profiles for values of $G r$.

the relative thermal buoyancy force values with respect to that of viscous hydrodynamic force enhanced the flow in the system. This is because the fluid flow gets warmer as it moves along the boundary layer surface thereby reduces the flow resistance forces which then result in an increase in the fluid flow rate. For low buoyancy effects, the maximum flow velocity occurs at the surface.

Figure 4 and Figure 5 represent the effect of a variational increase in the values of non-uniform heat generation parameters $\lambda$ and $\lambda^{*}$ on the temperature distributions. Follow from the Figures, was a rise in $\lambda$ and $\lambda^{*}$ values increased the temperature profiles. This resulted to an increase in the thermal boundary layer thickness which in turn reduces the amount of heat that diffuse out of the system and thereby causing a reduction in fluid dragging forces and enhances the velocity of the fluid particles in the system. Hence, it increases the fluid velocity and temperature profile. 


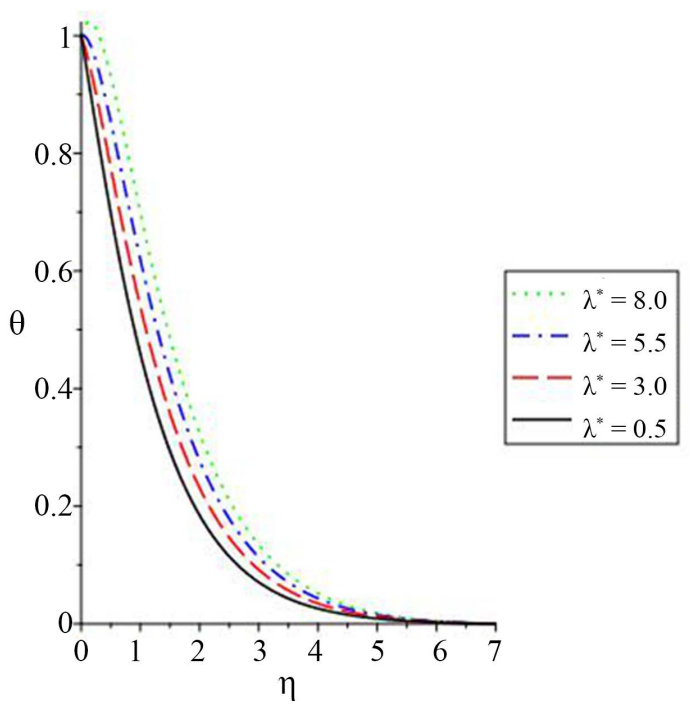

Figure 4. Temp. Profiles for values of $\lambda^{*}$.

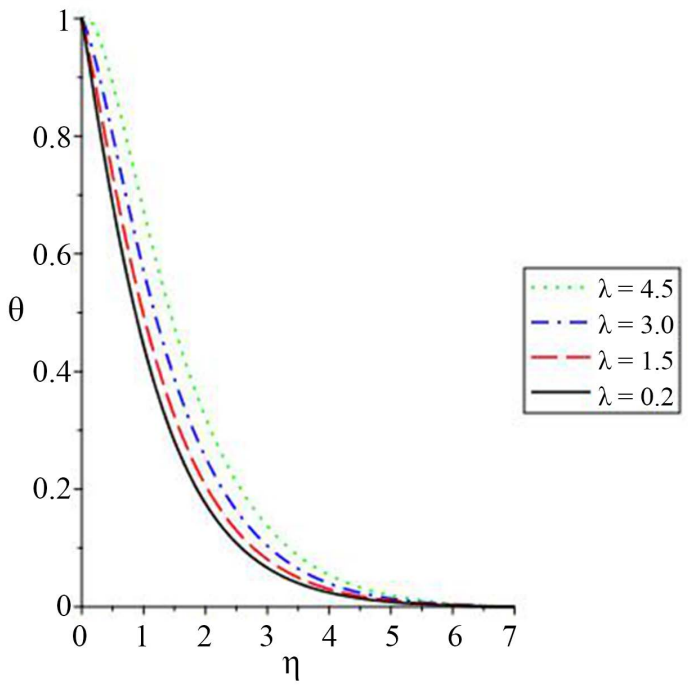

Figure 5. Temp. Profiles for various values of $\lambda$.

The reaction of the ratio of rates on fluid velocity field is presented in Figure 6. It is noticed that the velocity profile is enhanced with a rise in the parameter $\gamma$. Boundary layer thickness increases for the values of $\gamma>0$ because the rate of stretching dominates the rate of free stream flow thereby increases the velocity profiles.

The effect of loss load $Z$ on fluid velocity is shown in Figure 7. It is seen from the graph that as the values of the parameter $Z$ is increasing there is a decrease in the velocity distribution. The fluid velocity retarded due to load loss in form of heat as a result of friction in viscous flow. The boundary layers to get thinner and causes heat loss to the surrounding thereby strengthen the fluid dragging forces that in turn reduces the fluid flow velocity profiles.

Figure 8 and Figure 9 show the behavior of curvature parameter $K$ on the 


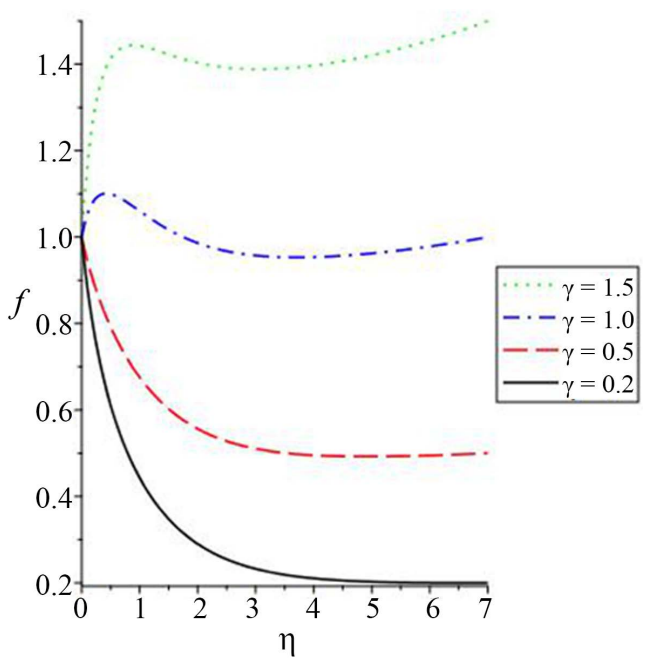

Figure 6. Velocity Profiles for various values of $\gamma$.

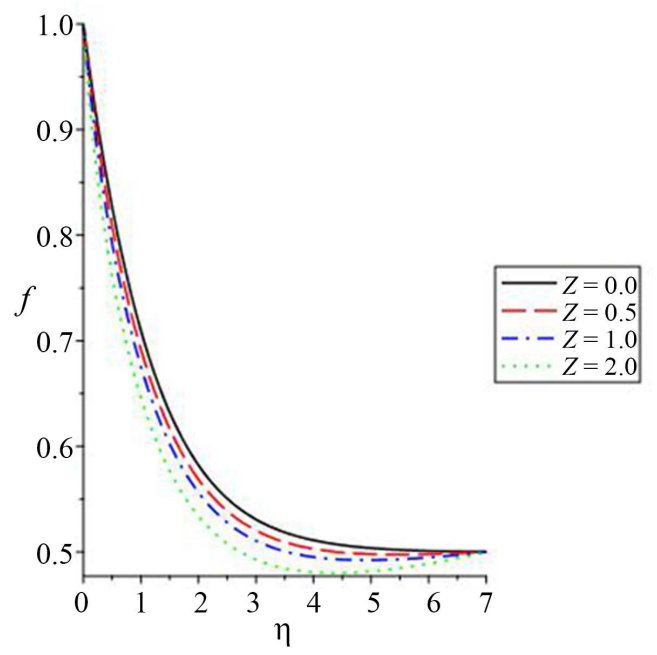

Figure 7. Velocity Profiles for values of $Z$.

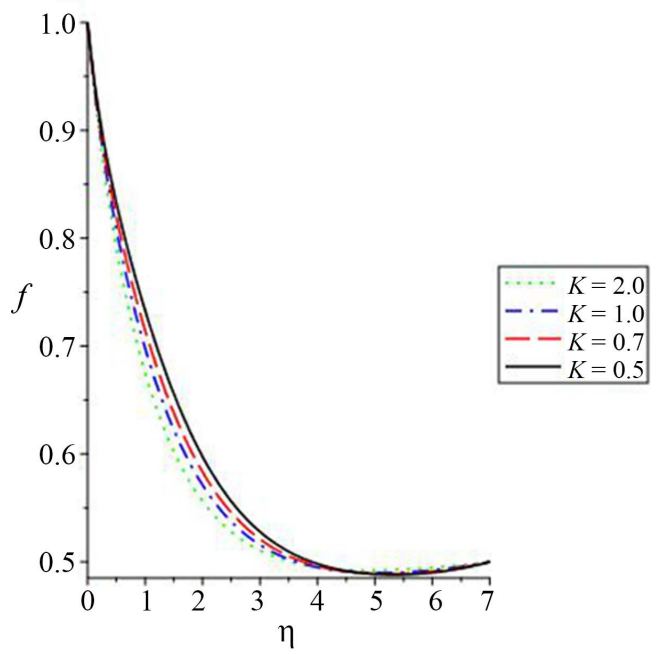

Figure 8. Velocity Profiles for various values $K$. 


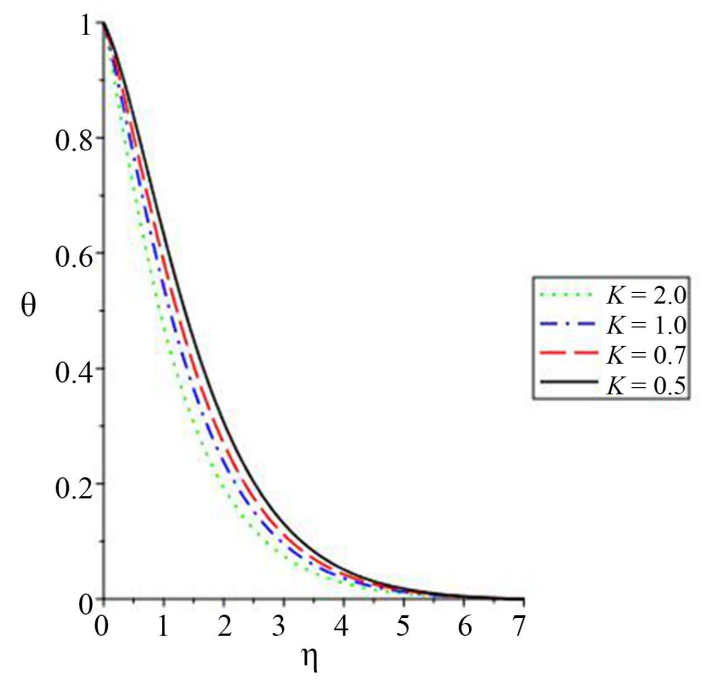

Figure 9. Temp. Profiles for various values $K$.

temperature and momentum field. It is obtained that velocity and temperature distribution has diminishing behavior near the cylinder surface while it increases far away from it. However, it is observed that thickness of the boundary layer reduces. Physically it is proved that larger values of curvature parameter $K$ reduce the cylinder radius. Consequently, the contact surface area of the cylinder with the flowing liquid and temperature decreases which provides large resistance to the motion of the fluid and increase the rate at which heat is diffuse out of the system thereby decreases the velocity and heat profiles.

The influence of diverse values of Prandtl number $\operatorname{Pr}$ on the temperature distribution is illustrated in Figure $10 \mathrm{It}$ is found that the temperature field reduces as increasing Pr values produces dampen effect on the profiles. Prandtl number is the ratio of momentum diffusivity to thermal diffusivity and it controls the relative thickness of the momentum and thermal boundary layers. Hence, increasing Pr implies a reduction in the thermal boundary layer thickness.

The influence of viscous dissipation parameter $E c$ on the temperature field is illustrated in Figure 11. Eckert number is the ratio of the kinetic energy of the flow to the boundary layer enthalpy difference. Kinetic energy is converted into internal energy by work done against the viscous fluid stresses. It is evident from the figures that an increase in the Eckert $E c$ number enhances the energy at any point in the flow and thereby causes the temperature profiles to increase.

Figure 12 represents the influences of the suction or injection parameter $f_{w}$ on the dimensionless momentum profiles. It is observed from Figure 12 that the suction parameter diminishes the velocity indicating the fact that suction stabilizes the boundary layer development, while the injection enhances the velocity at the boundary layer indicating that injection supports the flow to penetrate more into the fluid.

The effect of radiation parameter $N$ on temperature distributions are displayed in Figure 13. It is evident from this figure that thermal radiation diminishes the 


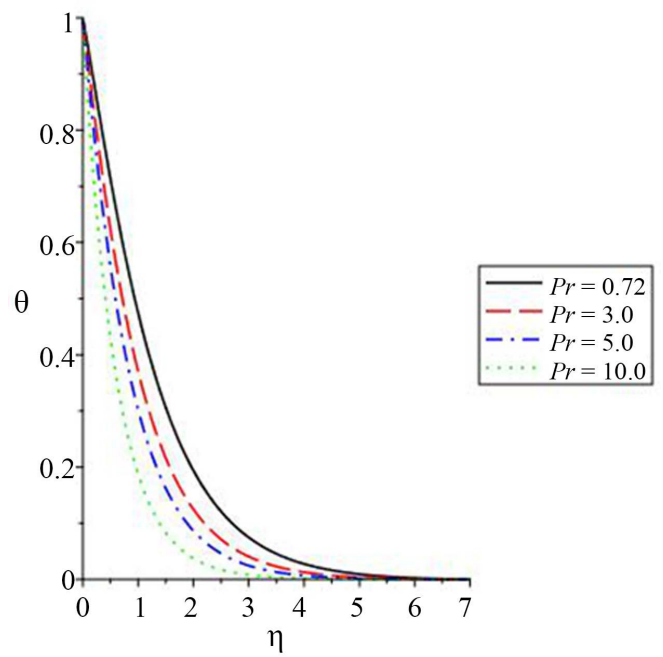

Figure 10. Temp. Profiles for various values Pr.

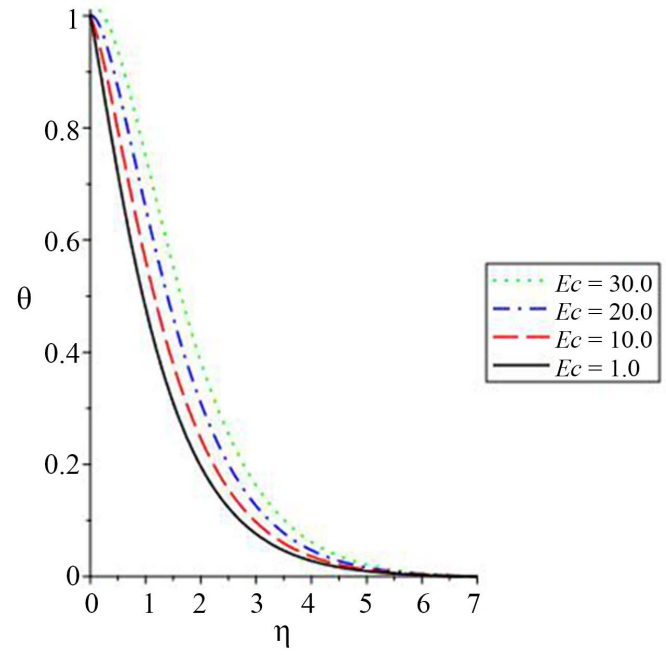

Figure 11. Temp. Profiles for various values $E c$.

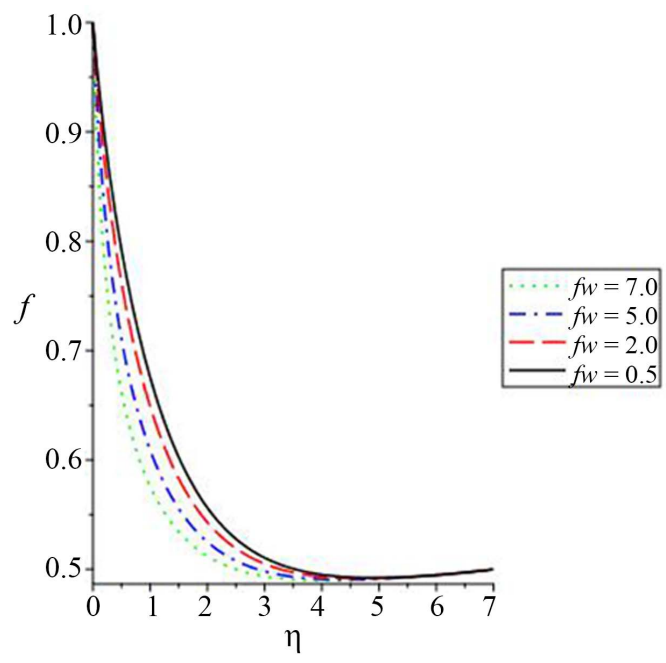

Figure 12. Velocity Profiles for various values $f_{w}$. 


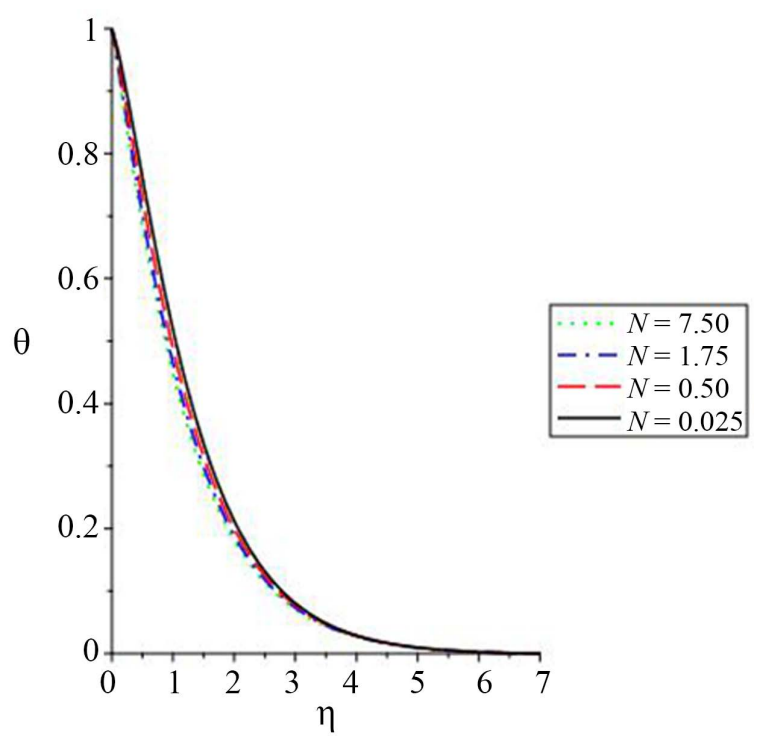

Figure 13. Temp. Profiles for various values $N$.

heat transfer. Hence, the flow temperature profiles decrease as $N$ increases. Thermal radiation is the relative contribution of heat conduction transfer to thermal radiation. Hence, an increase in the magnitude of radiation parameter $N$ reduces the thermal boundary layer. The reason for this is that the divergence of the radiative heat flux qr decreases as the Rosseland mean absorption coefficient $\delta$ increases. Thus, the rate of radiative heat transfer to the fluid decreases and then causing the temperature of the fluid to decline. Therefore, to have the cooling process at a faster rate, $N$ should be enhancing. The effect of thermal radiation becomes more significant as $N \rightarrow \infty$.

Figure 14 expresses the influence of viscous dissipation parameter $E \mathcal{C}$ on the energy gradient. The profiles decline close to the plate as result of friction due to a viscosity that drags the flow down but rises as the heat dissipation increases as it moves a distance away from the boundary layer towards the free stream due to enhancement in the rate of heat transfer within the fluid flow.

The action of the ratio of momentum diffusivity to thermal diffusivity on the Nusselt number is displayed in Figure 15. The rate at which heat is transferred out of the system is noticed to be decreasing as Prandtl number Pr is increased around the plate but heat diffuse out of the system continue to be rising gradually as it keeps distance from the plate surface at $\eta>1$ due decline in thermal boundary layer thickness which leads to decrease in the temperature gradient profiles.

\section{Conclusion}

A model for characterization of magneto hydrodynamic (MHD) flow through a confined cylinder was achieved when validated with some existing scholars' research work. However, formulated model was transformed to nonlinear coupled differential equation and solved using shooting technique of fourth-order 


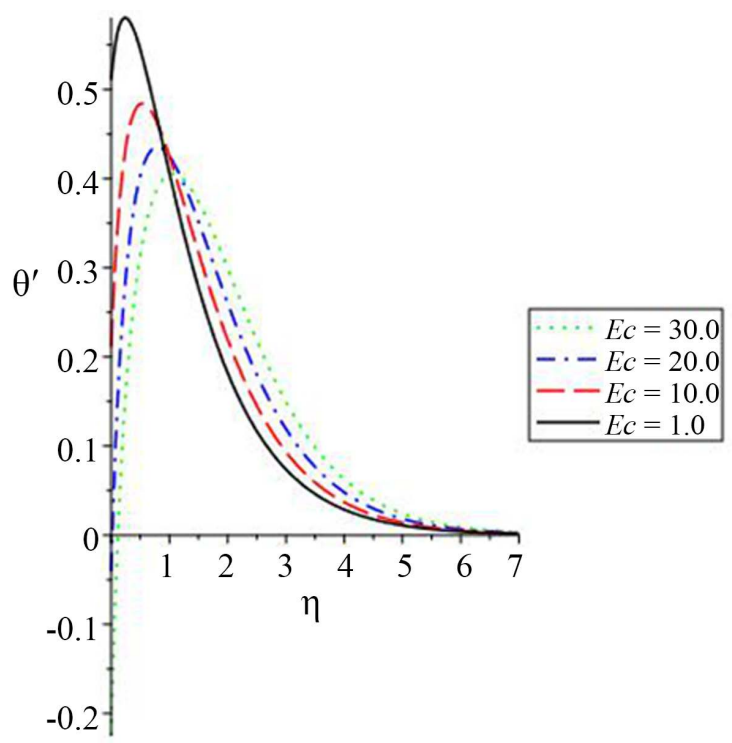

Figure 14. Nusselt number on various values $E c$.

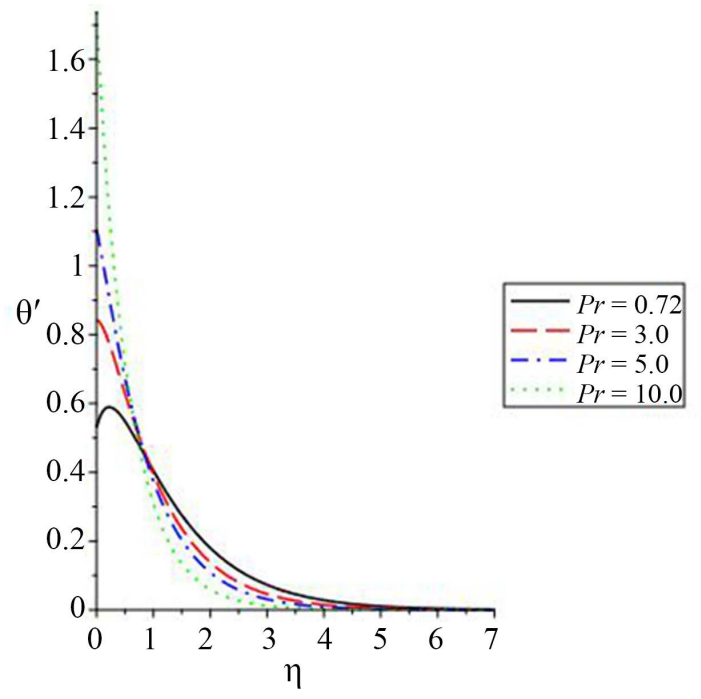

Figure 15. Nusselt number on various values Pr.

Runge-kutta method. In this study, Maple (2016) software is used for the computational analysis of the work with computer code developed for Equations (12)-(14). The effect of embedded fluid parameters on the flow momentum and energy balance was investigated and exhibited graphically. Likewise, the responses of the skin friction and Nusselt number to variation increase in some germane parameters are also illustrated graphically and quantitatively discussed. The detailed physical understanding is the scientific advantage of the analysis and discussion is furnished in Sections 3 and 4, which could not have been treasured easily from a global, overall solution of all the equations in one goal. The outcome of this analysis is very important because fluid flow past a permeable surface is useful in the areas of engineering, science and several transport processes in nature. 


\section{Acknowledgements}

The authors appreciate the Tetfund Nigeria (Lagos state Polytechnic, Ikorodu, Lagos) for her financial contribution towards this publication.

\section{Conflicts of Interest}

The authors declare no conflicts of interest regarding the publication of this paper.

\section{References}

[1] Hannes, A. (1942) Existence of Electromagnetic-Hydrodynamic Waves. Nature, 150, 405-406. https://doi.org/10.1038/150405d0

[2] Dada, M.S. and Salawu, S.O. (2017) Analysis of Heat and Mass Transfer of an Inclined Magnetic Field Pressure-Driven Flow Past a Permeable Plate. Applications and Applied Mathematics, 12, 189-200.

[3] Chamkha, A.J. (1998) Unsteady Hydromagnetic Flow and Heat Transfer from a Non-Isothermal Stretching Sheet Immersed in a Porous Medium. International Communications in Heat and Mass Transfer, 25, 899-906.

https://doi.org/10.1016/S0735-1933(98)00075-X

[4] Abel, M.S., Mahesha, N. and Tawade, T. (2009) Heat Transfer in a Liquid Film over an Unsteady Stretching Surface with Viscous Dissipation in Presence of External Magnetic Field. Applied Mathematical Modelling, 33, 3430-3441. https://doi.org/10.1016/j.apm.2008.11.021

[5] Altintas, A. and Ozkol, I. (2015) Magnetohydrodynamic Flow of Liquid-Metal in Circular Pipes for Externally Heated and Non-Heated Cases. Journal of Applied Fluid Mechanics, 8, 507-514. https://doi.org/10.18869/acadpub.jafm.67.222.22862

[6] Mukhopadhyay, S. (2009) Effect of Thermal Radiation on Unsteady Mixed Convection Flow and Heat Transfer over a Porous Stretching Surface in Porous Medium. International Journal of Heat and Mass Transfer, 52, 3261-3265.

https://doi.org/10.1016/j.ijheatmasstransfer.2008.12.029

[7] Alam, M.S., Hossain, S.M.C., Ahammad, M.U. and Hanif Sarkar, M.A. (2013) Dufour-Soret Effects on Radiative Free Convective Flow along Aninclined Permeable Stretching Sheet Embedded in a Porous Medium with Chemical Reaction. Jagannath University Journal of Science, 2, 39-54.

[8] Loganathan, P., Kannan, M. and Ganesan, P. (2011) Thermal Radiation Effects on MHD Flow over a Moving Semi-Infinite Vertical Cylinder. Int. Journal of Math. Analysis, 5, 257-274.

[9] Salawu, S.O. and Fatunmbi, E.O. (2017) Dissipative Heat Transfer of Micropolar Hydromagnetic Variable Electric Conductivity Fluid Past Inclined Plate with Joule Heating and Non-Uniform Heat Generation. Asian Journal of Physical and Chemical Sciences, 2, 1-10. https://doi.org/10.9734/AJOPACS/2017/31889

[10] Tsai, R., Huang, K.H. and Huang, J.S. (2008) Flow and Heat Transfer over an Unsteady Stretching Surface with a Non-Uniform Heat Source. I International Communications in Heat and Mass Transfer, 35, 1340-1343. https://doi.org/10.1016/j.icheatmasstransfer.2008.07.001

[11] Chen, C.H. (2008) Effects of Magnetic Field and Suction/Injection on Convection Heat Transfer of Non-Newtonian Power-Law FLUIDS past a Power-Law Stretched Sheet with Surface Heat Flux. International Journal of Thermal Sciences, 47, 954-961. https://doi.org/10.1016/j.ijthermalsci.2007.06.003 
[12] Sravanthi, C.S. and Leela Ratnam, A. (2014) Radiation Effects on MHD Boundary Layer Slip Flow along a Stretching Cylinder with Cross-Diffusion. International Journal of Engineering and Management Research, 4, 41-49.

[13] Hooshmand, P., Gatabi, H.R., Bagheri, N., Pirzadeh, I., Hesabi, A., Jamalabadi, M.Y.A. and Oveisi, M. (2017) Numerical Study of the Magnetic Field Effects on the Heat Transfer and Entropy Generation Aspects of a Power-Law Fluid over an Axisymmetric Stretching Plate Structure. Entropy (MDPI), 19, 1-22. https://doi.org/10.3390/e19030094

[14] Shahri, F. and Alireza, H.N. (2017) Investigation of Magnetohydrodynamics Flow and Heat Transfer in the Presence of a Confined Square Cylinder Using SM82 Equations. Thermal Science, 21, 889-899. https://doi.org/10.2298/TSCI140313048F

[15] Crane, L.J. (1975) Boundary LAYER flow Due to a Stretching Cylinder. Zeitschrift für angewandte Mathematik und Physik ZAMP, 26, 619-622.

https://doi.org/10.1007/BF01594034

[16] Wang, C.Y. (1988) Fluid Flow Due to a Stretching Cylinder. Physics of Fluids, 31, 466-468. https://doi.org/10.1063/1.866827

[17] Ishak, A., Nazar, R. and Pop, I. (2008) Uniform Suction/Blowing Effect on Flow and Heat Transfer Due to a Stretching Cylinder. Applied Mathematical Modelling, 32, 2059-2066. https://doi.org/10.1016/j.apm.2007.06.036

[18] Wan Zaimi, W.M., Ishak, A. and Pop, I. (2013) Unsteady Viscous Flow over a Shrinking Cylinder. Journal of King Saud University, 25, 143-148.

https://doi.org/10.1016/j.jksus.2012.11.005

[19] Kalteh, M., Ghorbani, S. and Khademinejad, T. (2016) Viscous Dissipation and Thermal Radiation Effects on the Magnetohydrodynamic (MHD) Flow and Heat Transfer over a Stretching Slender Cylinder. Journal of Applied Mechanics and Technical Physics, 57,463-472. https://doi.org/10.1134/S002189441603010X

[20] Salawu, S.O. and Amoo, S.A. (2016) Magnetohydrodynamics Effects on Radiative and Dissipative Heat Transfer near a Stagnation Point with Variable Viscosity and Thermal Conductivity. Computer Information Systems, 7, 51-62.

[21] Ishak, A. and Nazar, R. (2009) Laminar Boundary Layer Flow along a Stretching Cylinder. European Journal of Scientific Research, 36, 22-29.

[22] Hayat, T., Qayyum, S., Alsaed, A. and Asghar, S. (2017) Radiation Effects on the Mixed Convection Flow Induced by an Inclined Stretching Cylinder with Non-Uniform Heat Source/Sink. PLoS ONE, 12, e0175584. https://doi.org/10.1371/journal.pone.0175584

[23] Vajravelu, K., Prasad, K.V. and Santhi, S.R. (2012) Axisymmetric Magnetohydrodynamic (MHD) Flow and Heat Transfer at a Non-Isothermal Stretching Cylinder. Applied Mathematics and Computation, 219, 3993-4005. https://doi.org/10.1016/j.amc.2012.10.034

[24] Alizadeh, R., Rahimi, A.B. and Najafi, M. (2016) Magnetohydrodynamic Unaxisymmet-Ric Stagnation Point Flow and Heat Transfer of a Viscous Fluid on a Stationary Cylinder. Alexandria Engineering Journal, 55, 37-49. https://doi.org/10.1016/j.aej.2016.01.016

[25] Mastroberardino, A. and Siddique, J.I. (2014) Magnetohydrodynamic Stagnation Flow and Heat Transfer toward a Stretching Permeable Cylinder. Advances in $\mathrm{Me}$ chanical Engineering, 14, 1-5. https://doi.org/10.1155/2014/419568

[26] Sharma, P.R. and Singh, G. (2009) Effects of Variable Thermal Conductivity and Source/Sink on MHD Flow near a Stagnation Point on a Linearly Stretching Sheet. 
Journal of Applied Fluid Mechanics, 2, 13-21.

[27] Mukhopadhyay, S. (2012) Mixed Convection Boundary Layer Flow along a Stretching Cylinder in Medium. Journal of Petroleum Science and Engineering, 96-97, 73-78. https://doi.org/10.1016/j.petrol.2012.08.006

[28] Pal, D. and Mondal, H. (2012) Non-Darcian Buoyancy Driven Heat and Mass Transfer over a Stretching Sheet in a Porous Medium with Radiation and Ohmic Heating. International Journal of Nonlinear Science, 14, 115-123.

[29] Pop, S.R., Grosan, T. and Pop, I. (2004) Radiation Effect on the Flow near the Stagnation Point of a Stretching Sheet. Technische Mechanik, 25, 100-106.

[30] Hayat, T., Anwar, M.S., Farooq, M. and Alseadi, A. (2014) MHD Stagnation Point Flow of Second Grade Fluid over Stretching Cylinder with Heat and Mass Transfer. International Journal of Nonlinear Sciences and Numerical Simulation, 15, 365-376. https://doi.org/10.1515/ijnsns-2013-0104 